\title{
Decentralized control of sound radiation using a high-authority/low-authority control strategy with anisotropic actuators
}

N. H. Schiller, R. H. Cabell, and C. R. Fuller

Acoustics'08 Paris

July 3, 2008 
This paper describes a combined control strategy designed to reduce sound radiation from stiffened aircraft-style panels. The control architecture uses robust active damping in addition to high-authority linear quadratic Gaussian (LQG) control. Active damping is achieved using direct velocity feedback with triangularly shaped anisotropic actuators and point velocity sensors. While active damping is simple and robust, stability is guaranteed at the expense of performance. Therefore the approach is often referred to as low-authority control. In contrast, LQG control strategies can achieve substantial reductions in sound radiation. Unfortunately, the unmodeled interaction between neighboring control units can destabilize decentralized control systems. Numerical simulations show that combining active damping and decentralized LQG control can be beneficial. In particular, augmenting the in-bandwidth damping supplements the performance of the LQG control strategy and reduces the destabilizing interaction between neighboring control units.

\section{Introduction}

Active control strategies designed to reduce broadband interior noise in commercial and general aviation aircraft have received a lot of attention in the past decade. Since a coherent broadband reference signal is rarely available, a common approach is to feed back signals from accelerometers or piezoelectric transducers to piezoceramic actuators integrated in the structure [1]. One particularly simple and robust control strategy is referred to as active damping. The popularity of this strategy is attributed to Balas [2], who showed that if the transducer pairs are matched, then any passive control law, such as negative rate feedback, will guarantee the unconditional stability of the closed-loop system. However, practical limitations inevitably limit the performance of the control system. For instance, real transducer pairs are never perfectly matched, which eliminates the passive property of the system at high frequencies. Therefore, the control gain must be limited to avoid spillover and stability problems. As a result, a considerable amount of research has focused on the development of "substantially collocated" actuator-sensor pairs.

In contrast, model-based control strategies such as linear quadratic Gaussian (LQG) control do not require matched transducer pairs. However, since the control strategy is model based, the performance of the controller depends on the fidelity of the model [3]. Poorly modeled dynamics can destabilize the closed-loop system. Despite this limitation, researchers have shown that model-based control strategies can be used to reduce sound radiation from relatively simple structural acoustic systems. However, significant implementation issues have to be addressed before these control strategies can be extended to large systems such as the fuselage of an aircraft [4]. For instance, centralized approaches typically require a high level of connectivity and are computationally intensive while decentralized strategies face stability problems caused by the unmodeled interaction between neighboring control units.

This paper contains a numerical study of both types of control strategies. The first section briefly describes the numerical model used in the investigation. The limitations of decentralized LQG control are then highlighted. In particular, decentralized control of coupled subsystems is shown to introduce unavoidable errors that limit closed-loop performance. Direct velocity feedback (DVF) is then discussed. Since this approach is most effective when the transducers are matched, triangularly shaped actuator and point sensor pairs are studied. In particular, we show that a triangularly shaped anisotropic actuator can be collocated (at least theoretically) with a point sensor. Finally the benefits of a combined LQG/DVF strategy are discussed.

\section{System studied}

The stiffened flat panel depicted in Fig. 1 was used to evaluate the control architectures considered in this work. While many academic studies use simply-supported or clamped plates, those models are not representative of the aircraft fuselage at low frequencies where the structural wavelengths are long and the motion of both the panel and stiffeners is important [5]. In addition, those simple models neglect the structural coupling between bays, which can destabilize decentralized control systems [6]. Since stringers typically span a larger distance between supports and are more flexible than ring frames, the numerical model used here consists of a flat clamped panel partitioned into two bays by a flexible stringer. The dimensions and physical properties of the two-bay structure are included in Table 1.

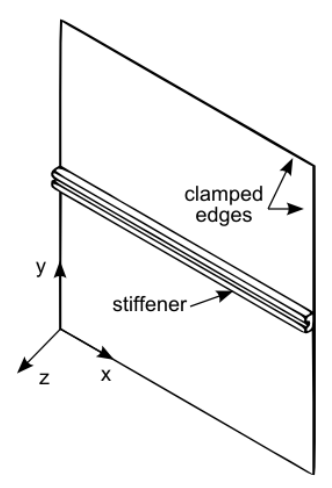

Figure 1: A diagram of the clamped, stiffened panel.

Table 1: Dimensions and physical properties of the two-bay model.

\begin{tabular}{lc}
\hline \hline Material & 6061-T6 Aluminum \\
Dimensions $\left(l_{x}, l_{y}, l_{z}\right)$ & $(0.476,0.380,0.0013) \mathrm{m}$ \\
Density & $2680 \mathrm{~kg} / \mathrm{m}^{3}$ \\
Young's modulus & $6.93 \times 10^{10} \mathrm{~Pa}$ \\
Poisson's ratio & 0.33 \\
Modal damping ratio & 0.01 \\
\hline \hline
\end{tabular}

The stiffened panel was driven by a spatially uncorrelated disturbance using 50 uniformly distributed random point loads. At the same time performance was evaluated in terms of radiated sound power. While additional details are omitted here, Schiller [7] provides a thorough description of the structural-acoustic model used in this study.

The sensor-actuator configurations and control architectures considered in this paper are illustrated in Fig. 2. The first 
control system, depicted in Fig. 2(a), used decentralized LQG controllers with center mounted piezoelectric patches and accelerometers. When integrated, the summed response from each set of accelerometers provided an estimate of the volume velocity of each bay. This transducer configuration was selected based on the controller/transducer complexity work performed by Gibbs et al. [8]. The second control system, depicted in Fig. 2(b), utilized negative rate feedback with triangularly shaped piezoelectric patches and point sensors positioned along the clamped vertical edges of the structure. The configuration was selected based on the work of Gardonio and Elliott [9], who showed that the triangular shape improves the stability bounds of the control system. This in turn allows higher feedback gains and therefore better performance. Finally, the combined control system depicted in Fig. 2(c) was studied. The combined strategy was implemented by incorporating both DVF and LQG control.

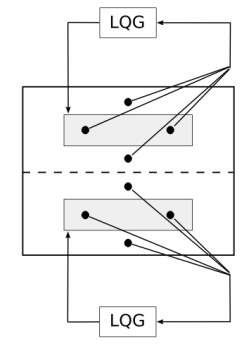

(a)

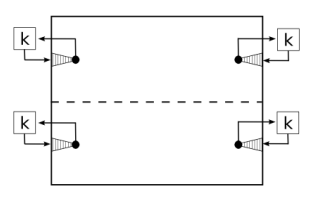

(b)

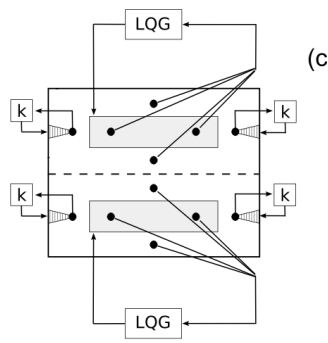

Figure 2: (a) Decentralized LQG configuration; (b) Closely located velocity sensors positioned at the tip of triangular actuators; (c) Combined control system.

\section{Decentralized linear quadratic con- trol}

Linear quadratic Gaussian (LQG) control was considered because it is relatively simple and well understood. In addition, past researchers have demonstrated that this type of control strategy can be used effectively for structural acoustic control [1]. LQG control is an optimal control strategy that uses minimum variance state estimates with an optimal state regulator. While the state regulator has guaranteed stability margins, the LQG controller can have arbitrarily poor stability margins due to errors in the state estimates. Since LQG controllers are not inherently robust, performance depends on the fidelity of the control model. Unfortunately decentralized control architectures inevitably introduce errors in the control model.

In the context of this work, decentralized control means that each controller is designed and implemented independently using local information. While the approach is scalable, the cross-coupling between subsystems introduces unmodeled dynamics in the control model [7]. Since LQG controllers are not inherently robust, it is easy to envision a scenario where local controllers could destabilize the coupled system. For example, Fig. 3 compares the radiated sound power from the stiffened panel with and without control. Two different controllers were considered. The thick red line shows the radiated sound power simulated using conservative LQG controllers while the thick dashed black line corresponds to a more aggressive design. This example illustrates the problem with decentralized LQG control. Conservative designs are often unable to meet performance goals while aggressive designs are susceptible to stability problems caused by the unmodeled interaction between neighboring controllers. For this system, the maximum integrated reduction in radiated sound power was limited to $1.6 \mathrm{~dB}$ using decentralized LQG control.

\section{Direct velocity feedback (DVF)}

Low-authority control strategies, such as direct velocity feedback (DVF), can be used to supplement or even replace highauthority LQG controllers. Unlike LQG control, direct velocity feedback is not model based. Therefore the approach is particularly attractive for decentralized control applications. If the transducer pair is matched, then the control system will be unconditionally stable. One way to generate substantially matched transducer pairs is to use small strain-based actuators along with point sensors. Unfortunately, the sensoractuator response is only positive real at low frequencies where the bending wavelength is larger than the dimensions of the actuator [10]. Therefore, negative rate feedback is only conditionally stable. Recently, Gardonio and Elliott [9] suggested that the performance of the control system could be improved using triangular actuators distributed around the perimeter of the panel. The triangular shape improves the stability bounds of the control system, which allows higher feedback gains and therefore better performance. However, triangularly shaped piezoelectric patches and point sensor pairs are not necessarily matched [9]. To understand why, note that triangularly shaped strain actuators can be modeled using transverse point loads at each vertex and bending moments along each edge [11] as shown in Fig. 4. The moment excitation along the base edge is defined as [9]

$$
m_{b}(t)=\frac{h_{s}}{2} e_{31} v_{c}(t)
$$

where $h_{s}$ is the combined thickness of the panel and the piezoelectric patch, $e_{31}$ is a piezoelectric material constant relating the electric field applied in the 3-direction to stress induced the 1-direction, and $v_{c}(t)$ is the applied voltage. Similarly, the moment excitation along the lateral edges is defined as

$$
m_{l}(t)=\frac{h_{s}}{2}\left(m^{2} e_{31}+e_{32}\right) v_{c}(t)
$$

where $m=b /(2 a)$ is the slope of the lateral edge, $b$ is the base of the triangle, and $a$ is its height. The point forces generated at the base vertices are

$$
f_{b}(t)=2 m \frac{h_{s}}{2} e_{31} v_{c}(t)
$$

while

$$
f_{t}(t)=-4 m \frac{h_{s}}{2} e_{31} v_{c}(t)
$$




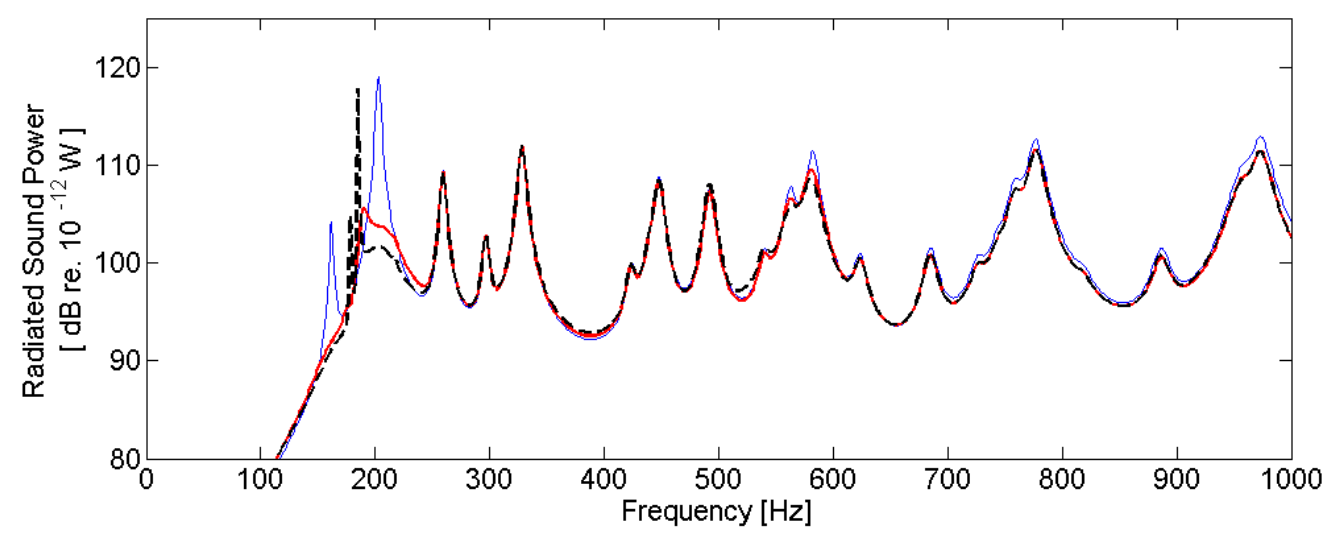

Figure 3: The radiated sound power from the structure before control (thin blue line), using conservative LQG controllers (thick red line), and using aggressive LQG controllers (dashed black line).

defines the point force at the tip of the triangular patch.

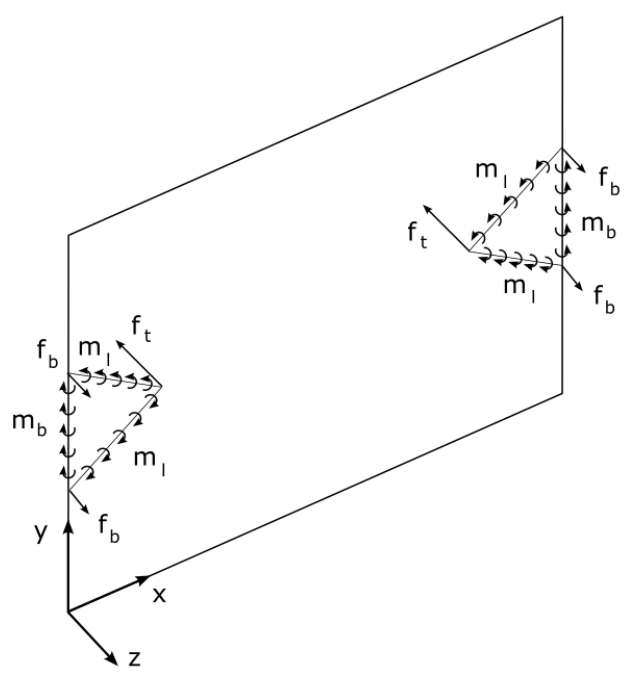

Figure 4: Triangular strain actuators represented in terms of point forces and line moments [adapted from Gardonio and Elliott [9]].

If the boundaries of the structure are clamped, then the point forces and line moments along the base of the actuator will not couple to the structural response. Therefore, a single point sensor placed at the vertex opposite the base edge can yield a substantially collocated frequency response. However, as the frequency increases and the bending wavelength approaches the dimensions of the actuator, the moments along the lateral edges couple more efficiently to the structural response than the point force at the tip of the actuator. As a result, the phase response tends to roll off at high frequencies. Therefore it is advantageous to eliminate the destabilizing line moments along the lateral edges of the actuator. This can be accomplished using anisotropic actuators that generate a tensile stress in one in-plane direction and a compressive stress in the other in-plane direction. One way to achieve this is to use an interdigitated electrode (IDE) pattern, as depicted in Fig. 5. While traditional piezoelectric actuators use uniform electrodes that apply the electric field through the thickness of the material (3-direction), the IDE allows the application of the electric field in the plane of the actuator (1-direction). The Macro-Fiber Composite (MFC) actuator [12] distributed by Smart Material Corp. is an example of a commercially available actuator with an IDE. In this case, the piezoelectric material constants are $e_{11}=11.9 \mathrm{~Pa} /(\mathrm{V} / \mathrm{m})$ and $e_{12}=-0.77 \mathrm{~Pa} /(\mathrm{V} / \mathrm{m})$.

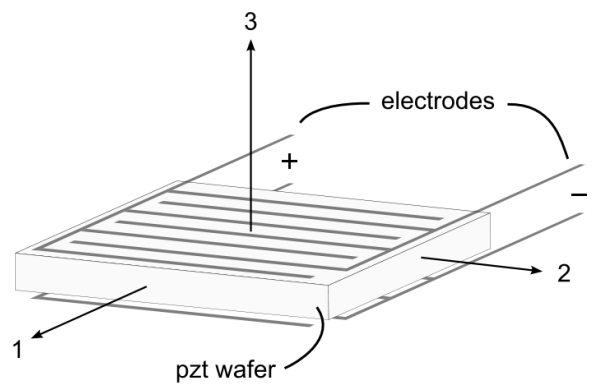

Figure 5: Interdigitated electrode pattern.

If a triangularly shaped anisotropic actuator is shaped such that

$$
m=\sqrt{-e_{12} / e_{11}}
$$

then the destabilizing line moments defined by Eq (2) will equal zero. In that case, the piezoelectric actuator will generate a point force at the tip of the triangle with negligible line moments along the lateral edges. For an MFC actuator, the lateral edge moments equal zero when the height of the triangle is approximately twice the width of the base. Therefore, the transducers considered in this work have base and height dimensions of $0.0325 \mathrm{~m}$ and $0.0635 \mathrm{~m}$, respectively.

For comparison, Fig. 6 shows simulated frequency response functions for an anisotropic triangular actuator and point sensor pair and for an ideal point force and point sensor pair. Notice that the two curves are essentially the same. The slight differences at high frequencies are primarily due to shaping errors caused by the numerical integration routine used to simulate the response of the piezostructure [7]. The additional mass and stiffness introduced by the piezoelectric patch also had a small impact on the high-frequency response. Note that while anisotropic triangular actuators and point sensors are promising, both shaping errors and highfrequency transducer dynamics will lead to phase accumulation when implemented in practice. In addition, the transducer pair will not necessarily be effective if placed along a flexible boundary [13]. 

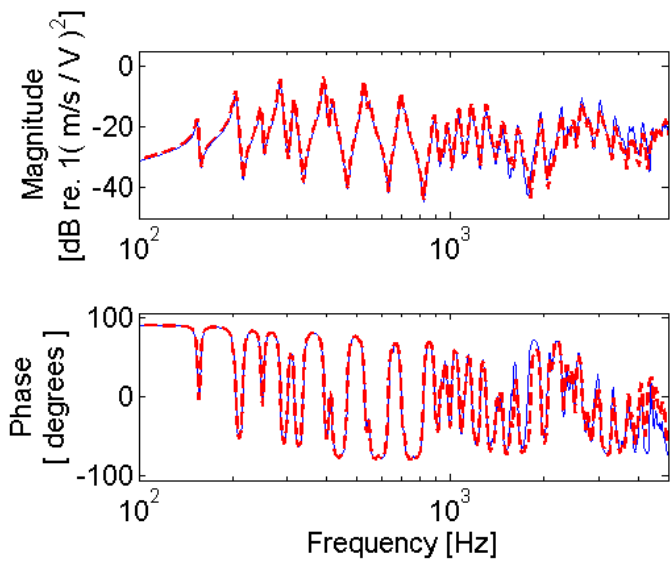

Figure 6: Frequency response functions for an anisotropic triangular actuator and point sensor pair (thin blue line), and an ideal point force input and point sensor pair (dashed red line).

Figure 7 shows the performance of the control system, depicted in Fig. 2(b), as a function of the feedback gain $k$. The thick black line demonstrates that excessive control gains can actually increase the sound power radiated from the structure. This occurs because large control gains pin the structure at the tip of each actuator, resulting in new lightly damped resonances [9, 14]. However when the optimal control gain is used, the system achieves a $4.8 \mathrm{~dB}$ integrated reduction in radiated sound power.

In practice, sound power measurements may not be available. Therefore another option is to use volume velocity estimates to determine the optimal control gain. The thin solid and dashed lines in Fig. 7 show the volume velocity estimates for each bay as a function of control gain. Each estimate was based on four summed and integrated accelerometers on each bay. The figure shows that any one of the measurements could be used to determine the optimal control gain. Since volume velocity estimates are also used for the LQG control system, the next step was to consider a combined control strategy.

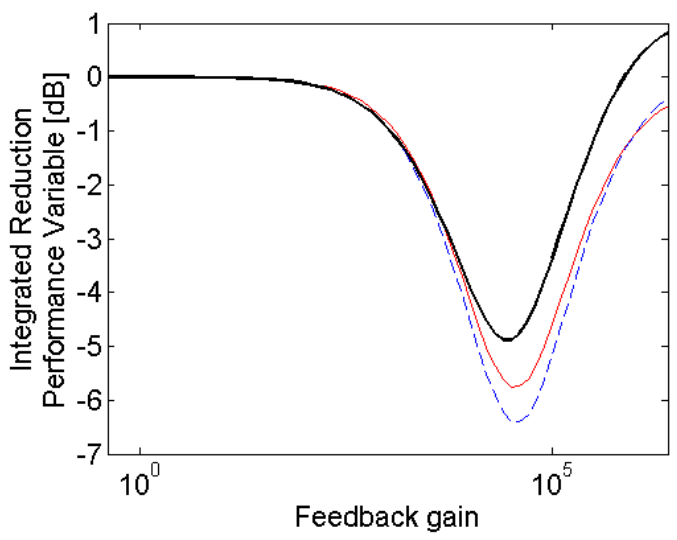

Figure 7: Integrated reduction in: radiated sound power (thick black line); volume velocity on the upper bay (thin red line); volume velocity on the lower bay (dashed blue line).

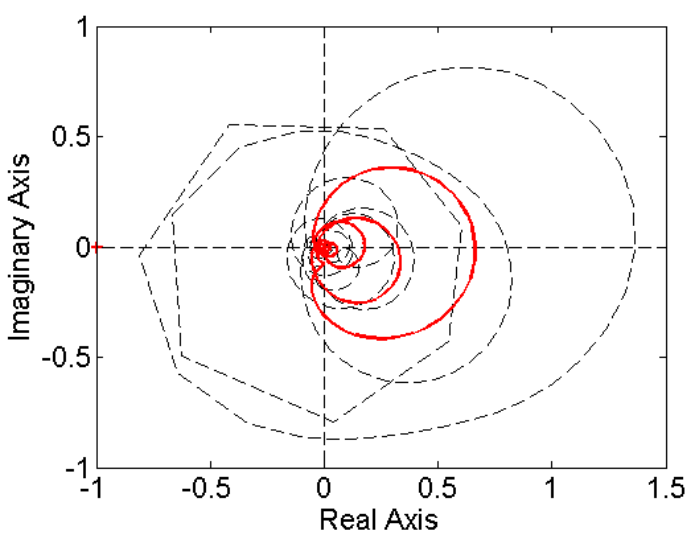

Figure 9: Nyquist plot of the sensor-controller-actuator transfer function using LQG control (thin dashed black line), and using DVF and LQG control (solid red line).

\section{Combined control strategy}

The combined LQG/DVF control strategy is shown in Fig. 2(c). The combined strategy was implemented by first incorporating DVF, and then identifying the system model and designing the LQG controllers. In this simulation, each low-authority controller was implemented in continuoustime, while the decentralized LQG control system was simulated in discrete-time at $3 \mathrm{kHz}$. The performance of the combined control system is shown with the solid red line in Fig. 8. The combined system achieved a $6.8 \mathrm{~dB}$ integrated reduction, which is significantly better than either control system achieved on its own. In addition, the combined system was more robust than LQG alone, as demonstrated by the Nyquist plot shown in Fig. 9. Including DVF improved the gain margin for the LQG system from $1.8 \mathrm{~dB}$ to $27 \mathrm{~dB}$.

\section{Concluding Remarks}

Decentralized controllers introduce unavoidable errors due to the unmodeled coupling between subsystems. One way to account for the modeling error is to use an inherently robust control strategy such as DVF. This requires matched transducer pairs, which can be formed using shaped anisotropic actuators along with point sensors. In particular, a triangularly shaped piezoelectric patch with an interdigitated electrode pattern can be used to eliminate the destabilizing line moments along the lateral edges of the transducer. Instead of using DVF alone, another option is to combine DVF with LQG control. This work shows that by augmenting the structural damping, DVF can stabilize a decentralized LQG control system. In addition, simulations show that a combined approach can outperform either individual strategy with a $6.8 \mathrm{~dB}$ integrated reduction in radiated sound power from 0 to $1000 \mathrm{~Hz}$.

\section{References}

[1] R.L. Clark, W.R. Saunders, G.P. Gibbs, Adaptive Structres, Dynamics and Control (Wiley, New York, 1998) 


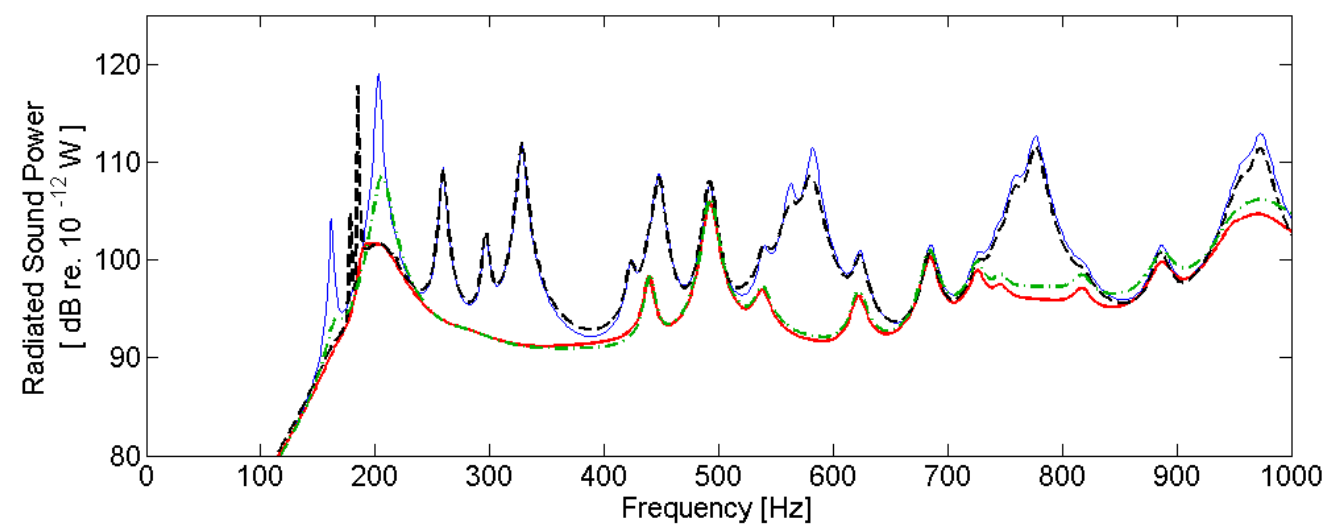

Figure 8: The radiated sound power from the structure before control (thin blue line), using decentralized LQG controllers (dashed black line), using DVF with the optimal feedback gain (dash-dotted green line), and using both DVF and LQG (solid red line).

[2] M.J. Balas, "Direct velocity feedback control of large space structures", J. Guid. Control, 2:252-253 (1979)

[3] J.C. Doyle, "Guaranteed margins for LQG regulators", IEEE Trans. Automatic Control, AC-23:756-757 (1978)

[4] R.H. Cabell, D.E. Cox, G.P. Gibbs, 'Interaction metrics for feedback control of sound radition from stiffened panels", Proceedings of the 44th AIAA/ASME/ASCE/AHS Structures, Structural Dynamics, and Material Conference 7-10 April, Norfolk, Virginia (2003)

[5] J.S. Mixson, J.F. Wilby, "Interior Noise", Aeroacoustics of Flight Vehicles, Theory and Practice (NASA, 1991)

[6] G.P. Gibbs, R.H. Cabell, "Simultanteous active control of turbulent boundary layer induced sound radiation from multiple aircraft panels", Proceedings of the 8th AIAA/CEAS Aeroacoustics Conference 17-19 June, Breckenridge, Colorado (2002)

[7] N.H. Schiller, "Decentralized control of sound radiation from periodically stiffened panels", $\mathrm{PhD}$ thesis, Virginia Tech, Blacksburg, Virginia (2007)

[8] G.P. Gibbs, R.H. Cabell, J. Juang, "Controller complexity for active control of turbulent boundary layer noise from panels", AIAA J. 42:1314-1320 (2004)

[9] P. Gardonio, S.J. Elliott, "Smart panels with velocity feedback control systems using triangularly shaped strain actuators", J. Acoust. Soc. Am., 117:2046-2064 (2005)

[10] P. Gardonio, E. Bianchi, S.J. Elliott, ”Smart panel with multiple decentralized units for the control of sound transmission. Part I: theoretical predictions", J. Sound Vib., 274:163-192 (2004)

[11] J.M. Sullivan, J.E. Hubbard, S.E. Burke, "Modeling approach for two-dimensional distributed transducers of arbitrary spatial distribution", J. Acoust. Soc. Am., 99:2965-2974 (1996)
[12] W.K. Wilkie, R.G. Bryant, J.W. High, R.L. Fox, R.F. Hellbaum, A. Jalkin, B.D. Little, P.H. Mirick, "Lowcost piezocomposite actuator for structural control applications", Proceedings of the SPIE 7th Annual International Symposium on Smart Structures and Materials, Newport Beach, California (2000)

[13] N.H. Schiller, C.R. Fuller, R.H. Cabell, "A highauthority/low-authority control strategy for coupled aircraft-style bays", Proceedings of Active-06, 18-20 September, Adelaide, Australia (2006)

[14] S.J. Elliott, P. Gardonio, T.C. Sors, M.J. Brennan, ”Active vibroacoustic control with multiple local feedback loops", J. Acoust. Soc. Am., 111:908-915 (2002) 